PSYCHOMETRIKA-VOL. 25 , No. 3

SEPTEMBER, 1960

\title{
ON A CONNECTION BETWEEN FACTOR ANALYSIS AND MULTIDIMENSIONAL UNFOLDING*
}

\author{
Clyde H. Coombs
}

UNIVERSITY OF MICHIGAN

AND

RICHARD C. KaO

\author{
PLANNING RESEARCH CORPORATION \\ LOS ANGELES, CALIFORNIA
}

\begin{abstract}
Given the preference ordering of each of a number of individuals over a set of stimuli, it is proposed that if the preference orderings are generated in a Euclidean space of $r$ dimensions which can be recovered by unfolding the preference orderings, then a factor analysis of the correlations between individual's preference orderings will yield a space of $r+1$ dimensions with the original $r$-space embedded in it, and the additional dimension will be one of social utility. The proposition is clearly shown to be satisfied by means of the Monte Carlo technique for both random and lattice stimuli in three dimensions and for two other examples with random stimuli in one and two dimensions.
\end{abstract}

The unfolding technique for preferential choice behavior $[7,8]$ is derived from a model of the following form. An individual, in making preferential choices among a set of alternatives, may be represented by a point in an $r$-dimensional Euclidean space, $E^{r}$, and correspondingly, each alternative may be represented by a point in the same space. The individual prefers one alternative to another if and only if the point corresponding to the preferred alternative is nearer to the point corresponding to the individual. To each point corresponds an $r$-tuple which is a set of measures on the dimensions spanning the space. These dimensions may be interpreted as psychological variables generating the preferences of the individuals, where the point corresponding to an individual is an ideal point representing a hypothetical alternative preferred to all other possible ones. Inconsistency

*The preparation of this paper was supported in part by a grant from the National Science Foundation and in part by Project MICHIGAN, a project of the University of Michigan in the field of Combat Surveillance sponsored by the Department of the Army. The contract (DA-36-039 ac 78801) is administered by the U. S. Army Signal Corps. The authors are indebted to L. A. Raphael, Caroline K. Tefft, and F. M. Goode for programming assistance, and to L. W. Staugas for providing other computer services during various stages of this study. 
of preferences, to be distinguished from intransitivity, may be generated by random variability in the locus of points [10].

According to the model, an individual's dominant preferences may be represented by a rank order scale of the alternatives given by the transitive set of stochastically determined pairwise preferences. Such a scale is called an $I$ scale and may be regarded as folding the space by picking it up at the ideal point and collapsing it into a line with the measure of the stimulus points on this line corresponding to their respective distances from the ideal point. Distinct ideal points generate distinct $I$ scales in this manner. With ordinal preference data such $I$ scales have a many-one mapping into equivalence classes corresponding to distinct rank order $I$ scales. The unfolding technique is the name given to the method for determining the number of dimensions and the rank order of the projections on the dimensions, and, in the case of one dimension, ordered metric information.

The problem of determining the dimensionality of a Euclidean space in which a set of $I$ scales may be unfolded was solved by Bennett [6] and the problem of determining the configuration of the points for both stimuli and individuals (called a Joint space) was solved by Hays [11].

The following problem naturally arises. Suppose one intercorrelated the individuals' $I$ scales and factor analyzed; what relation would the factorial solution have to the $E^{r}$ assumed to have generated the preferences?

\section{The Proposition}

Consider the simple case of a one-dimensional latent attribute generating the preferences of individuals over a set of alternatives. The ideal points of the individuals and the points for the alternatives are all points on a line, a Joint scale. To avoid sampling fluctuations, assume the stimulus points are dense and that the two sets of points range over the same segment of the line.

Consider the $I$ seale of an individual $(A)$ at the extreme left end of the scale and that of another individual very close to him. Clearly, their preference orderings will be almost identical and will correlate close to plus one. The $I$ scale for individual $A$ will correlate progressively less with $I$ scales of other individuals as they are farther removed from him on the Joint scale. In fact, the correlation will be zero between individual $A$ and the median individual in the distribution, and will ultimately be minus one between him and the individual at the extreme opposite end of the scale. The median individual will have correlations ranging from close to plus one with those individuals near him on either side, to zero with the individuals at either end.

Clearly, if each individual is represented by a unit vector from a common origin and the correlation between individuals by the cosine of the angle between the corresponding vectors, the configuration corresponding to the correlation matrix will be a semicircle with the individuals corresponding to a fan of vectors such that the vector of the median individual projects verti- 
cally upward and orthogonal to the vectors of the two extreme individuals which form an angle of 180 degrees. The order of the termini of the vectors on the arc would correspond exactly to the order of the corresponding points on the original line.

If one factor analyzes such a configuration by the method of principal components, the first dimension would be the original line which generated the preferential choices; the second dimension would be the vector of the median individual on the line. On the latter dimension the projections of individual points are in reverse order with respect to how closely each is to all the others on the line. In another context, this second dimension is called a social utility [9]. The higher the projection, the more nearly that point best represents all the other points in the sense of being nearest to them all.

If we consider the case of a two-dimensional latent attribute space generating the preferential choices, we now have two superimposed bivariate distributions-one for individuals and one for stimuli. If one considers the correlation of the $I$ scale of an individual on the rim of this space with other individuals, it seems reasonable that the correlations will progressively decrease through zero to minus one as one approaches an individual across the space from him, and that the median individual on the plane will correlate non-negatively with everyone. The configuration generated by the set of unit vectors is now a hemisphere in three dimensions, with the median individual represented by a unit vector perpendicular to the plane in which the vectors of all individuals on the rim of the plane lie. If such were the case, a factor analysis would yield three dimensions, with the third principal component again corresponding to a social utility and the first two dimensions representing the original space which generated the preferential choices.

While not as intuitively obvious, we may generalize this proposition to a space of $r$ dimensions in which we would expect the configuration corresponding to the correlation matrix to be a semihypersphere in $r+1$ dimensions; the $(r+1)$ th principal component would be a social utility and the first $r$ dimensions would correspond to the original space.

This proposition was first conjectured by the first author but later more fully studied by the second using the Monte Carlo technique. Any attempt to realize the idealized version of the proposition would necessarily lead to some distortion, the matching of the two being sensitive to the density of stimulus points and the joint distribution of stimulus and individual points and to the measure used for the correlation between two individuals' $I$ scales. In practice only a finite number of stimulus points can be used so the working definition of a genotypic space is the chosen finite set of stimulus points. The theorem which is conjectured is this: given $m$ arbitrary points in $E^{n}$, then they lie in an $r$-subspace of $E^{n}$ if and only if with probability 1 , the rank of the product moment correlation matrix approaches $r+1$ as the number of stimulus points approaches infinity. 


\section{Imbedding of Genotypic Space into Factor Space}

In order to test the plausibility of the proposition discussed above under rather general and varying conditions, several problems were constructed and explored, of which two related ones in three dimensions play the major role. These will be presented first.

Three sets of 15 random numbers are taken to represent the coordinates of 15 individuals in $E^{3}$, and another three sets of 30 random numbers, those of 30 stimuli in the same space (Tables 1 and 2). (All numbers [14] were

TABLE 1

Coordinates of Individual points in $\mathrm{E}^{3}$

\begin{tabular}{cccc}
\hline & & & \\
\hline 01 & -0.47883 & -0.12812 & $c$ \\
\hline 02 & -0.20438 & -0.40540 & 0.30109 \\
03 & -0.49558 & 0.23608 & 0.26483 \\
04 & 0.41039 & -0.42816 & -0.18766 \\
05 & -0.45173 & 0.54625 & -0.29035 \\
06 & 0.08085 & 0.29372 & 0.41746 \\
07 & -0.26920 & -0.34540 & -0.04339 \\
08 & 0.27289 & 0.32257 & 0.07160 \\
09 & 0.05593 & -0.13210 & -0.36360 \\
10 & 0.15816 & 0.00408 & -0.33086 \\
11 & 0.40540 & -0.27578 & -0.34882 \\
12 & -0.46175 & -0.39914 & -0.23506 \\
13 & 0.25472 & 0.54289 & 0.01397 \\
14 & 0.30705 & -0.05145 & -0.32123 \\
15 & 0.41614 & 0.22003 & 0.48096 \\
& & & 0.49106 \\
\hline
\end{tabular}

first taken to be seven-place decimal fractions and computations carried out in this manner, but rounded to five places after the completion of the study.) Since all numbers were decimal fractions, the Joint space for both individuals and stimuli is, by definition, a cube in $E^{3}$ with length of its sides equal to 2 and center at the origin, called the basic cube. A third set of points is taken to represent a second set of stimulus points, these being the 64 lattice points of a "grid" contained in the basic cube. On each dimension the points take on one of the four values $-.6,-.2,+.2,+.6$, yielding $4^{3}=64$ points. For simplicity, we shall distinguish the two sets of stimulus points by calling them random stimuli and lattice stimuli, respectively. The motivation for taking the latter is twofold: (i) to see if an increase in the number of stimuli used would yield a better fit to the idealized situation, and (ii) to test if the model were feasible with quite arbitrary selection of stimulus points, random as well as nonrandom. 
TABLE 2

Coordinates of Random Stimulus Points in $E^{3}$

\begin{tabular}{|c|c|c|c|}
\hline & a & b & c \\
\hline 01 & 0.03991 & -0.40188 & 0.28193 \\
\hline 02 & -0.38555 & -0.34414 & 0.32386 \\
\hline 03 & $0.17540^{\circ}$ & 0.10461 & 0.39510 \\
\hline 04 & $-0.326+3$ & -0.52861 & 0.27699 \\
\hline 05 & -0.24122 & -0.30231 & -0.10274 \\
\hline 06 & 0.30532 & 0.21704 & -0.35075 \\
\hline 07 & -0.03788 & 0.42402 & 0.56623 \\
\hline$O B$ & 0.48228 & -0.07405 & -0.36409 \\
\hline 09 & -0.32960 & 0.53845 & 0.57620 \\
\hline 10 & -0.19322 & -0.57260 & 0.07399 \\
\hline 11 & -0.11220 & -0.47744 & -0.14454 \\
\hline 12 & 0.31751 & -0.48893 & 0.07481 \\
\hline 13 & -0.30934 & 0.16993 & 0.27499 \\
\hline 14 & 0.22888 & 0.33049 & -0.35902 \\
\hline 15 & -0.41849 & -0.08337 & -0.46850 \\
\hline 16 & -0.46352 & 0.36898 & 0.14013 \\
\hline 17 & -0.11087 & -0.48297 & 0.56303 \\
\hline 18 & -0.52701 & -0.19019 & 0.39904 \\
\hline 19 & 0.57275 & 0.32486 & 0.45134 \\
\hline 20 & -0.20857 & 0.01839 & 0.37239 \\
\hline 21 & 0.15633 & 0.07629 & -0.18637 \\
\hline 22 & -0.38688 & 0.43625 & -0.05327 \\
\hline 23 & 0.25163 & -0.11692 & 0.43253 \\
\hline 24 & 0.36815 & 0.25624 & -0.53342 \\
\hline 25 & -0.04515 & 0.06345 & -0.13574 \\
\hline 26 & 0.14387 & -0.00008 & -0.29593 \\
\hline 27 & 0.51321 & 0.55306 & -0.44989 \\
\hline 28 & 0.05466 & 0.18711 & 0.52152 \\
\hline 29 & -0.39528 & -0.16120 & 0.04737 \\
\hline 30 & -0.07586 & -0.04235 & 0.16894 \\
\hline
\end{tabular}

The Euclidean distances of each individual from all the stimuli (random or lattice) are computed and these measures provide an $I$ scale for the individual, which is a ratio scale rather than an ordinal scale. The product moment correlations are then computed between each pair of individuals' $I$ scales yielding two correlation matrices, one for the random stimuli $M_{r}$, (Table 3) and one for the lattice stimuli $M_{l}$ (Table 4). These correlation matrices, with unity in the diagonal are then factored by the method of principal components. Two different subroutines (IBM 704 and RAND JOHNNIAC) were used independently to duplicate all computations. The 


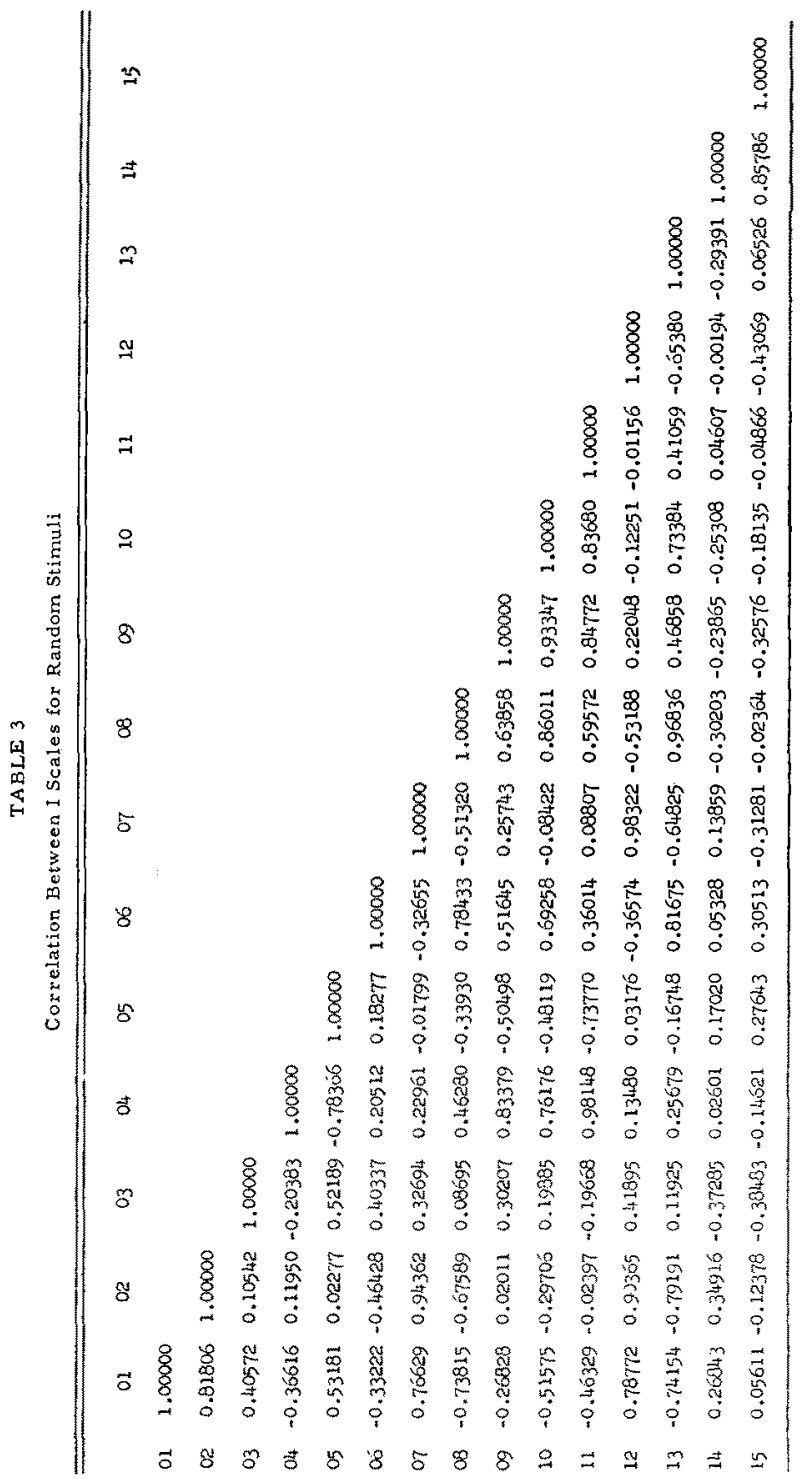

numbers from these two sources agree to seven decimal places except for signs, i.e., a characteristic vector from one subroutine may be the reflection of another from the other subroutine.

The characteristic values, $\lambda_{i}$, for the two correlation matrices are given in Table 5. It can be seen that a sharp drop in the magnitude of the characteristic value occurs after the fourth one. We take, therefore, the first four columns of the factor matrices (Tables 6 and 7 ) as factor loadings or co- 

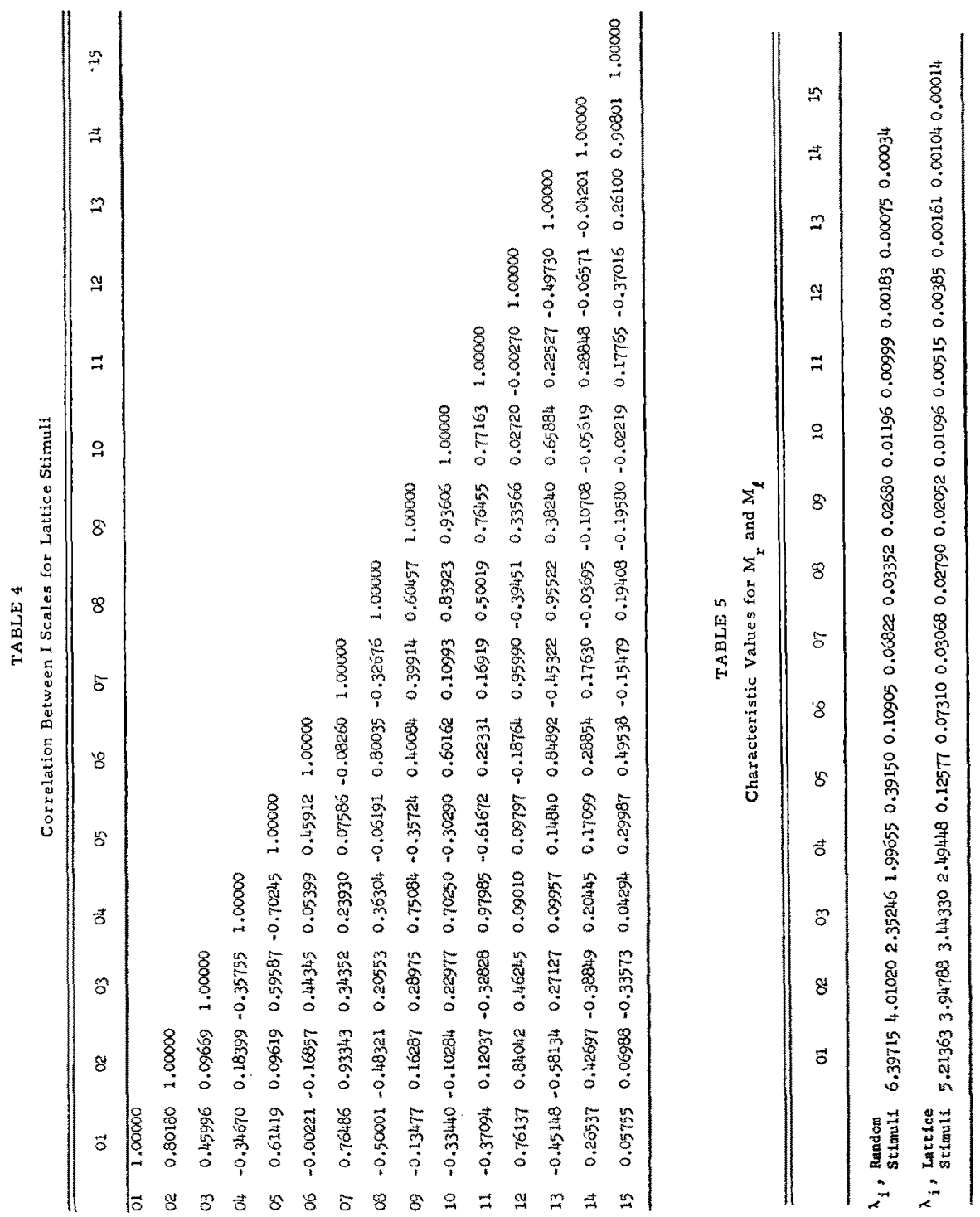

ordinates of the 15 individuals in four dimensions. The statistical theory for testing the number of significantly positive characteristic roots of a sample correlation matrix has yet to be worked out (cf. [1], p. 330). Our investigation lends some convincing evidence that such a theory can be developed [cf. 1, $2,3,4,5,12]$.

Two crucial questions arise. First, how are the original coordinates of 
TABLE 6

Principal Companents Factor Loatings for Random Stimuli

\begin{tabular}{|c|c|c|c|c|}
\hline & Ol & O2 & 03 & 04 \\
\hline & 6.39715 & 4.01020 & 2.35246 & 1.99655 \\
\hline 01 & 0.85262 & 0.27021 & .0 .22809 & 0.33375 \\
\hline 02 & 0.69980 & 0,6́2891 & 0.17248 & 0.26119 \\
\hline$O_{3}$ & 0.08552 & 0.25101 & -0.92805 & 0.20117 \\
\hline 04 & $=0.57057$ & 0.70975 & 0.38252 & 0.06207 \\
\hline 05 & 0.47920 & -0.48049 & -0.56888 & 0.39698 \\
\hline os & -0.68648 & -0.08115 & $-0.4 \mathbf{1} 818$ & 0.54391 \\
\hline 07 & 0.55962 & 0.78716 & -0.04066 & 0.21914 \\
\hline$\infty 8$ & -0.96190 & 0.00826 & -0.20677 & 0.03215 \\
\hline$\infty$ & -0.63852 & 0.73509 & -0.16018 & 0.13697 \\
\hline 10 & -0.85590 & 0.46673 & -0.15916 & 0.12525 \\
\hline 12 & -0.69323 & 0.60280 & 0.34346 & 0.12922 \\
\hline 12 & 0.59545 & 0.76164 & -0.16490 & 0.11234 \\
\hline 13 & -0.92659 & $-0,28631$ & -0.18912 & 0.26799 \\
\hline 14 & 0.25163 & -0.14168 & 0.57725 & 0.74380 \\
\hline 15 & -0.101312 & -0.56050 & 0.39376 & 0.76243 \\
\hline
\end{tabular}

TABLE ?

Principal Components Factor Loadings for Lattice Stimuli

\begin{tabular}{|c|c|c|c|c|}
\hline & OI & 02 & 03 & 04 \\
\hline & 5.21353 & 3.94788 & 3.44330 & 2.49448 \\
\hline 01 & 0.72719 & 0,46805 & -0.45787 & 0.14273 \\
\hline$\infty 2$ & 0.53410 & 0.78105 & -0.01001 & 0.29306 \\
\hline 03 & 0.10687 & 0.26099 & -0.76577 & -0.54484 \\
\hline $\mathrm{OH}_{4}$ & -0.59226 & 0.60106 & 0.48881 & 0.15722 \\
\hline 05 & 0.37333 & -0.17152 & -0.87300 & 0.12318 \\
\hline 06 & -0.58528 & 0.09476 & -0.77412 & 0.19804 \\
\hline 07 & 0.43858 & 0.89902 & -0.09576 & 0.00533 \\
\hline 08 & -0.92210 & 0.02215 & -0.35550 & -0.07667 \\
\hline$\infty$ & -0.61730 & 0.73370 & -0.02764 & -0.25316 \\
\hline 10 & -0.83504 & 0.50160 & -0.12971 & -0.16607 \\
\hline 11 & -0.69580 & 0.55692 & 0.36150 & 0.23753 \\
\hline 12 & 0.51236 & 0.81177 & -0.10147 & -0.22775 \\
\hline 13 & -0.81839 & -0.18864 & -0.51428 & -0.05858 \\
\hline 14 & 0.00093 & 0.18049 & -0.10485 & 0.97188 \\
\hline 15 & -0.18760 & -0.12257 & -0.27787 & 0.92650 \\
\hline
\end{tabular}


the individual points in three dimensions related to their factor loadings in four dimensions? Second, what is the significance of the "extra" dimension obtained?

According to the proposition, the configuration corresponding to the correlation matrix is a set of unit vectors in $E^{4}$ whose projections in a subspace $E^{3}$ orthogonal to the median individual will faithfully reproduce the configuration of the individual points in the original genotypic space. Hence, the first question can be settled if we show that Table 1 can be "imbedded" into Table 6 and into Table 7 .

To this end, Tucker's method of congruence is used [15]. His coefficient of congruence, $Q_{r}$, is similar to a product moment correlation between the loadings on factor $r$ in the factor space and those in the original space.The values of $Q_{r}$ for each of the three original dimensions as recovered by the two factor analyses are given in Table 8 . The congruence appears reasonably

TABLE 8

Congruence of Original Dimensions with Factor Space

\begin{tabular}{lccc}
\hline & $\mathbf{r}_{1}$ & $\mathbf{r}_{2}$ & $\mathbf{r}_{3}$ \\
\hline$Q_{\mathbf{r}}$, Random Stimuli & .99492 & .97699 & .98790 \\
$Q_{\mathbf{r}}$, Lattice Stimuli & .99154 & .98254 & .99687 \\
\hline
\end{tabular}

high and a good fit of the original configuration of individual points into a three-dimensional subspace of the factor space is possible.

\section{The Extra Dimension in the Factor Space}

According to the proposition, the genotypic space can be imbedded in the factor space; the factor space will have an additional dimension and the projection of a point on this extra dimension will be related to how close each point was to all the other points in the genotypic space. The first two parts of the proposition have been sustained by the results reported above and it remains now to test the last part.

The projection of each vector on the extra dimension of the factor space is readily given knowing the length of the vector in the factor space of four dimensions and its reduced length in the three-dimensional subspace that corresponds to the original genotypic space.

The average distance of any point from all the others in the original genotypic space is readily obtained from Table 1 . The smaller the average distance of a point from all the others the nearer the point lies to the median of the population and hence the higher its projection on the extra dimension 
of the factor 'space. The Spearman rank order correlations between average distances in genotypic space (ordered from smallest to largest) with projections on the extra dimension (ordered from largest to smallest) is .723 and .896 for random and lattice stimuli respectively, significant at the .005 level. It follows, therefore, that there is reasonable evidence for answering in the affirmative both questions which led us to include a second set of stimulus points in the three-dimensional problem.

Two more problems were run to test the proposition when the genotypic space is of dimension 1 or 2 . For this purpose, only one set of stimulus points was retained by pairing off the first column in Table 1 against that in Table 2 , or the first two columns in Table 1 against those in Table 2. Euclidean distances between individuals and random stimuli in 1 and 2 dimensions were first computed and then the correlation matrices of individuals over stimuli, which were factored by the method of principal components. Only a summary of the results are presented here. The first five (and largest) characteristic values for the one-dimensional case and the two-dimensional case are presented in Table 9 .

TABLE 9

Characteristic Values for the One- and Two-Dimensional Genotypic Space

\begin{tabular}{lccccc}
\hline \hline & 1 & 2 & 3 & 4 & 5 \\
\hline One-Dimensiona 1 & 12.02233 & 2.70781 & 0.13125 & 0.10578 & 0.01797 \\
Two-Dimensiona 1 & 7.38998 & 4.81759 & 2.36532 & 0.24135 & 0.07356 \\
\hline
\end{tabular}

A sharp drop in the magnitude of the characteristic value occurs after the second for the one-dimensional case and after the third for the twodimensional case, indicating that the factor space for preferences had one additional dimension beyond the genotypic space which generated the preferences. Again Tucker's method is used for maximal congruence and the $Q$, for the one-dimensional case is 0.976 and for the two-dimensional case are 0.989 and 0.986 for the first and second dimensions respectively. Spearman rank order correlations between average distance of an individual's point from all the others in the genotypic space and the projection of the individual on the extra dimension were 0.761 and 0.669 , significant at the 0.005 level, for the one- and two-dimensional cases respectively.

\section{Discussion}

We recapitulate briefly the main results of the preceding two sections. A Joint space is taken with both individuals and stimuli as points in it. An 
$I$ scale of preferences over the stimuli is constructed for each individual by taking the Euclidean distances of these stimuli from the individual's ideal point. Correlating individuals' $I$ scales gives rise to a matrix of correlations which are factored by the method of principal components. In each problem, the dimension of the factor space is noticeably one higher than the original genotypic space. But, the configuration of the individual points in the original genotypic space can be faithfully reproduced in a hyperplane of the factor space. The rank orders of the projections of the individual vectors on the extra dimension correlate highly in reverse order with those of their genotypic space. These results are obtained when the Joint space is of different dimensions and the stimulus points are quite arbitrarily chosen.

There are several aspects which need to be discussed because of their relevance to the practical application of the propositions tested here. In any practical application there would be two sources of error or distortion, one of which is present in this study. The first is that the basic data would normally consist of rank order preference scales rather than the actual distances to stimulus points. This means that the product moment correlation can only be approximated. The second is that the distribution of stimulus points relative to that for the individuals can distort the factor space. This is most obviously evident in the one-dimensional case in which the stimuli that lie between two individuals tend to produce negative correlation between their preference orderings and the stimuli that lie outside of them tend to produce positive correlation. Clearly if the density of the stimulus points between two individuals is unusually high or low, the correlation between their preferences will be biased toward negative or positive correlation, and they will appear in the factor space as farther apart or nearer together than in the genotypic space.

A further aspect relevant to practical application is that in the real case one arrives first at the factor space and seeks the genotypic space. This requires determining the extra dimension in the factor space, with no prior knowledge of the genotypic space, and then rotating it out in order to work with just the genotypic space that remains. The following argument suggests how this may be done.

Our model states that all individual vectors in the genotypic space are "blown up" into unit vectors whose termini lie on a semihypersphere bounded by a hyperplane containing the genotypic space. In this process, the distance in the genotypic space of an individual from the median individual is changed by a monotone transformation into the distance on the semihypersphere between the termini of the unit vectors representing these individuals in the factor space. Therefore, the rank orders of the distances of all individuals from the median individual will not be affected. If a rotation about the origin is made of all individual vectors in the factor space, these rank orders still remain invariant. This means that we may determine the 
social utility dimension in the factor space in exactly the same manner as we do in the genotypic space. That is, we use the coordinates of individuals in the factor space and find the median individual accordingly. The social utility dimension is then passed through this individual and the projections of other individuals on this dimension can be computed. By our observation above, the rank orders of all individuals from this median individual should correlate highly with those in the original genotypic space.

\section{Summary and Conclusions}

A model called the unfolding technique for analyzing preferential choice data assumes that individuals and stimuli may be represented by points in a Fuclidean space of $r$ dimensions and that an individual's preference ordering of the stimuli reflects the order of their increasing distance from his position in the space. Such a preference ordering is called an $I$ scale. Given the $I$ scales of a number of individuals, methods are available for determining the dimensionality of the space and the configuration of points in the space.

On the other hand, correlations between the preference orderings of individuals could be computed and the resulting correlation matrix factor analyzed. Naturally arising then is the question of what the relation would be between the genotypic space which gives rise to the preference orderings, and is recovered by the unfolding technique, and the space obtained by multidimensional factor analysis.

A heuristic argument was presented for the following propositions:

(i) if the genotypic space is Euclidean with $r$ dimensions, the factor space will have $r+1$ dimensions;

(ii) the genotypic space can be imbedded in the factor space;

(iii) the additional dimension in the factor space will be a social utility dimension in the sense that the nearer a point is to all the other points in the genotypic space the higher its projection is on this extra dimension in the factor space.

The problem was studied by the Monte Carlo technique. Three sets of 15 random numbers were taken as the coordinates of 15 individuals in $E^{3}$ and three sets of 30 random numbers, those of 30 stimuli in the same space. A second set of stimuli points was taken as the 64 lattice points of a cube 2 units on a side with center at the origin. Given this genotypic space, preference scales of individuals were computed for the random and for the lattice stimuli, correlation matrices between individual's preferences were obtained and factored by the method of principal components. This procedure was carried out for both sets of stimuli with $r=3$ and with only the random stimuli with $r=1$ and $r=2$.

Tucker's method was used to test for congruence of the genotypic and factorial spaces. All three propositions were confirmed for both random and lattice stimuli with some slight superiority in favor of the lattice stimuli. 
This could be due to the larger number of lattice stimuli or the regularity of their distribution or both.

The social utility dimension in the factor space was discussed including a possible method for isolating it.

The most general practical consequence of this development is that the methods of multiple factor analysis are revealed to be suitable for the discovery of the latent attribute variables underlying preferences after the social utility dimension has been removed, with the qualification that there will be some sensitivity to the density and the distribution of stimulus points in the space. A recent study by MacRae [13] is a case in point and the theory and technique developed here would have been useful in that study.

\section{REFERENCES}

[1] Anderson, T. W. An introduction to multivariate statistical analysis. New York: Wiley, 1958. Ch. V.

[2] Bartlett, M. S. Tests of significance in factor analysis. Brit. J. Psychol., Statist. Sec., $1950,3,77-85$.

[3] Bartlett, M. S. The effect of standardization on a $\chi^{2}$-approximation in factor analysis. Biometrika, 1951, 38, 337-344.

[4] Bartlett, M. S. A further note on tests of significance in factor analysis. Brit. $J$. Psychol., Statist. Sec., 1951, 4, 1-2.

[5] Bartlett, M. S. Factor analysis in psychology as a statistician sees it. Uppsala symposium on psychological factor analysis. Uppsala, Sweden: Almqvist and Wiksell, 1953. Pp. 23-34.

[6] Bennett, J. F. and Hays, W. L. Multidimensional unfolding: determining the dimensionality of ranked preference data. Psychometrika, 1960, 25, 27-44.

[7] Coombs, C. H. Psychological scaling without a unit of measurement. Psychol. Rev., $1950,57,145-158$.

[8] Coombs, C. H. A theory of psychological scaling. Engin. Res. Inst. Bull, No. 34. Ann Arbor: Univ. Michigan Press, 1952.

[9] Coombs, C. H. Social choice and strength of preferenees. In R. M. Thrall, C. H. Coombs, and R. L. Davis (Eds.), Decision processes. New York: Wiley, 1954. Pp. 69-86.

[10] Coombs, C. H. Inconsistency of preferences in psychological measurement. $J$. exp. Psychol, 1958, 55, 1-7.

[11] Hays, W. L. and Bennett, J. F. Multidimensional unfolding: determining configuration from complete rank order preference data. Psychometrika. (in press)

[12] Kendall, M. G. and Smith, B. B. Factor analysis. J. roy statist. Soc. $(B), 1950,12$, 60-94.

[13] MacRae, D., Jr. A factorial analysis of political preferences. Revue Francaise de Science Politique, 1958, 8, 95-109.

[14] RAND Corporation. A million random digits with 100,000 normal deviates. Glencoe, Illinois: Free Press, 1955.

[15] Tucker, L. R. A method for synthesis of factor analysis studies. Princeton: Educ. Test. Serv. Res. Bull. No. 984, 1951.

Manuscript received 3/25/59

Revised manuscript received 11/19/59 